\title{
Análise Numérica para Um Problema de Difusão Termoelástica em Domínios Não-Cilíndricos
}

\author{
Rodrigo L. R. Madureira ${ }^{1}$ \\ Instituto de Matemática, Programa de Pós-Graduação em Informática (PPGI), UFRJ, Rio de \\ Janeiro, RJ \\ Mauro Antonio Rincon ${ }^{2}$ \\ Instituto de Matemática, Departamento de Ciência da Computação, UFRJ, Rio de Janeiro, RJ
}

\begin{abstract}
Resumo. Estudamos o problema de valor inicial e de contorno para um sistema unidimensional de equações lineares de difusão termoelástica em domínio não-cilíndrico. São mostrados três métodos para obtenção da solução numérica aproximada: acoplado, desacoplado com preditor-corretor e desacoplado. A simulação numérica é feita através do método dos elementos finitos no espaço e diferenças finitas no tempo. Além disso, são mostradas as taxas de convergência dos métodos aplicados.
\end{abstract}

Palavras-chave. Difusão termoelástica, Domínio não-cilíndrico, Elementos finitos, Diferenças finitas

\section{Introdução}

A difusão pode ser definida como um fenômeno de penetração, desde regiões de alta concentração até regiões de baixa concentração. O conceito de difusão está ligado ao de transferência de massa impulsionado por diferenças de concentração de diferentes regiões do material.

Problemas em domínios não-cilíndricos têm sido tratados por muitos autores e em várias direções. A existência e unicidade do problema é conhecido na literatura através dos trabalhos de M. Aouadi (ver [1-4]). Rincon et al. [5] analisaram o aspecto numérico do problema termoelástico em domínios não-cilíndricos.

\section{Problema original no domínio não-cilíndrico}

Considere o problema na forma homogênea:

\footnotetext{
${ }^{1}$ rodrigo.rangel@nce.ufrj.br

${ }^{2}$ rincon@dcc.ufrj.br
} 


$$
\begin{array}{r}
\rho \frac{\partial^{2} u}{\partial t^{2}}-\alpha \frac{\partial^{2} u}{\partial x^{2}}+\gamma_{1} \frac{\partial \theta}{\partial x}+\gamma_{2} \frac{\partial P}{\partial x}=0, \text { em } \widehat{Q} \\
c \frac{\partial \theta}{\partial t}+d \frac{\partial P}{\partial t}-k \frac{\partial^{2} \theta}{\partial x^{2}}+\gamma_{1} \frac{\partial^{2} u}{\partial x \partial t}=0, \text { em } \widehat{Q} \\
d \frac{\partial P}{\partial t}+\eta \frac{\partial \theta}{\partial t}-\hbar \frac{\partial^{2} P}{\partial x^{2}}+\gamma_{2} \frac{\partial^{2} u}{\partial x \partial t}=0, \text { em } \widehat{Q}
\end{array}
$$

com as seguintes condições de fronteira:

$$
u=\theta=P=0, \text { em } \widehat{\Sigma}
$$

e condições iniciais:

$$
u(x, 0)=u_{0}(x), \frac{\partial u}{\partial t}(x, 0)=u_{1}(x), \theta(x, 0)=\theta_{0}(x), P(x, 0)=P_{0}(x),-K(0)<x<K(0)
$$

onde $\widehat{Q}$ é o domínio não-cilíndrico, contido no $\mathbb{R}^{2}$, definido por:

$$
\widehat{Q}=\left\{(x, t) \in \mathbb{R}^{2} ; x=K(t) y, y \in\right]-1,1[, t \in] 0, T[\}
$$

com fronteira lateral

$$
\widehat{\Sigma}=\bigcup_{0<t<T}\{\{-K(t) \times\{t\}\} \cup\{K(t) \times\{t\}\}\}
$$

onde a função real $K(t)$ e as constantes $\alpha, \beta, \gamma$ e $k$ satisfazem certas condições:

(i) $K \in C^{2}([0, T] ; \mathbb{R}) \operatorname{com} K_{0}=\min _{0 \leq t \leq T} K(t)>0$

(ii) $\left|K K^{\prime \prime}\right|<c K^{2}$, onde $c>0$.

(iii) Existe uma constante positiva $K_{1}$, tal que $1-\left[K^{\prime}(t) y\right]^{2}>K_{1}$,

$0 \leq t \leq T,-1 \leq y \leq 1$.

(iv) $\rho, \alpha, \gamma_{1}, \gamma_{2}, k$ e $\hbar$ são estritamente positivas.

(v) Assuma que as constantes positivas $c, d$ e $\eta$ satisfazem

$$
c \eta-d^{2}>0
$$

O teorema de existência e unicidade a seguir do problema (1) pode ser encontrado nos trabalhos de Moncef Aouadi [1-4].

Teorema 2.1. Suponha que as hipóteses (i)-(v) são válidas. Então, com os dados iniciais $u_{0} \in H_{0}^{1}(-1,1), u_{1} \in L^{2}(-1,1), \theta \in L^{2}(-1,1), P \in L^{2}(-1,1)$

o problema envolvendo as equações 1-3 possui uma única solução fraca para u, $\theta$ e $P$. 


\section{Problema equivalente no domínio cilíndrico}

Note que quando $(x, t)$ varia em $\widehat{Q}$, o ponto $(y, t)$, com $y=K^{-1}(t) x$, varia no cilindro $Q=]-1,1[\times] 0, T[$. Assim, a função $\tau: \widehat{Q} \rightarrow Q$, dada por $\tau:(x, t) \rightarrow(y, t)$, é um difeomorfismo. Sob as mudanças de variáveis $v(y, t)=u(\underbrace{K(t) y}_{x}, t), \phi(y, t)=\theta(\underbrace{K(t) y}_{x}, t)$, $\psi(y, t)=P(\underbrace{K(t) y}_{x}, t)$, obtemos o problema equivalente:

$$
\mid \begin{aligned}
& \rho \frac{\partial^{2} v}{\partial t^{2}}-\rho \frac{\partial}{\partial y}\left(a_{1}(y, t) \frac{\partial v}{\partial y}\right)+2 \rho b_{2}(y, t) \frac{\partial^{2} v}{\partial y \partial t}+\rho a_{3}(y, t) \frac{\partial v}{\partial y} \\
& +(\rho-\alpha) b_{1}(t) \frac{\partial^{2} v}{\partial y^{2}}+\gamma_{1} a_{2}(t) \frac{\partial \phi}{\partial y}+\gamma_{2} a_{2}(t) \frac{\partial \psi}{\partial y}=0 \text { em } Q \\
& c \frac{\partial \phi}{\partial t}+c b_{2}(y, t) \frac{\partial \phi}{\partial y}+d \frac{\partial \psi}{\partial t}+d b_{2}(y, t) \frac{\partial \psi}{\partial y}+\gamma_{1} a_{2}(t) \frac{\partial^{2} v}{\partial y \partial t} \\
& +\gamma_{1} \frac{\partial}{\partial y}\left(b_{3}(y, t) \frac{\partial v}{\partial y}\right)-k b_{1}(t) \frac{\partial^{2} \phi}{\partial y^{2}}=0 \text { em } Q \\
& d \frac{\partial \psi}{\partial t}+d b_{2}(y, t) \frac{\partial \psi}{\partial y}+\eta \frac{\partial \phi}{\partial t}+\eta b_{2}(y, t) \frac{\partial \phi}{\partial y}+\gamma_{2} a_{2}(t) \frac{\partial^{2} v}{\partial y \partial t} \\
& +\gamma_{2} \frac{\partial}{\partial y}\left(b_{3}(y, t) \frac{\partial v}{\partial y}\right)-\hbar b_{1}(t) \frac{\partial^{2} \psi}{\partial y^{2}}=0 \mathrm{em} Q \\
& v=\phi=\psi=0, \mathrm{em} \Sigma \\
& v(y, 0)=v_{0}(y), \frac{\partial v}{\partial t}(y, 0)=v_{1}(y), \phi(y, 0)=\phi_{0}(y) \\
& \psi(y, 0)=\psi_{0}(y),-1<y<1
\end{aligned}
$$

onde:

$$
\begin{gathered}
a_{1}(y, t)=\frac{1}{K(t)^{2}}-\left(\frac{K^{\prime}(t) y}{K(t)}\right)^{2} ; a_{2}(t)=\frac{1}{K(t)} ; a_{3}(y, t)=-\frac{K^{\prime \prime}(t) y}{K(t)} ; \\
b_{1}(t)=\frac{1}{K(t)^{2}} ; b_{2}(y, t)=-\frac{K^{\prime}(t) y}{K(t)} ; b_{3}(y, t)=-\frac{K^{\prime}(t) y}{K(t)^{2}}
\end{gathered}
$$

\section{Sistema aproximado}

Seja $T>0$. Denotamos por $V_{m}$ o subespaço gerado por $w_{1}, w_{2}, \ldots, w_{m}$, onde $\left(w_{i}\right)_{i \in \mathbb{N}} \in$ $V_{m}$. Para cada $m \in \mathbb{N}$, consideramos as seguintes soluções aproximadas $v_{m}, \phi_{m}, \psi_{m}$ representadas por: 


$$
v_{m}(y, t)=\sum_{i=1}^{m} \lambda_{i}(t) w_{i}(y) ; \phi_{m}(y, t)=\sum_{i=1}^{m} \sigma_{i}(t) w_{i}(y) ; \psi_{m}(y, t)=\sum_{i=1}^{m} \xi_{i}(t) w_{i}(y)
$$

Definindo as seguintes matrizes:

$$
\begin{gathered}
M_{i j}=\left(w_{i}, w_{j}\right) ; N_{i j}=\left(\frac{\partial w_{i}}{\partial y}, \frac{\partial w_{j}}{\partial y}\right) ; L_{i j}=\left(\frac{\partial w_{i}}{\partial y}, w_{j}\right) ; \\
S_{i j}(t)=\left(a_{1}(y, t) \frac{\partial w_{i}}{\partial y}, \frac{\partial w_{j}}{\partial y}\right)=\sum_{e=1}^{m} a_{1}^{e}(t) N_{i j}^{e} ; U_{i j}(t)=\left(b_{2}(y, t) \frac{\partial w_{i}}{\partial y}, w_{j}\right)=\sum_{e=1}^{m} b_{2}^{e}(t) L_{i j}^{e} ; \\
V_{i j}(t)=\left(a_{3}(y, t) \frac{\partial w_{i}}{\partial y}, w_{j}\right)=\sum_{e=1}^{m} a_{3}^{e}(t) L_{i j}^{e} ; W_{i j}(t)=\left(b_{3}(y, t) \frac{\partial w_{i}}{\partial y}, \frac{\partial w_{j}}{\partial y}\right)=\sum_{e=1}^{m} b_{3}^{e}(t) N_{i j}^{e}
\end{gathered}
$$

e substituindo as soluções aproximadas na formulação variacional de (7), obtemos o seguinte sistema de equações diferenciais ordinárias no tempo:

$$
\mid \begin{aligned}
& \rho M \ddot{\lambda}(t)+2 \rho U^{T}(t) \dot{\lambda}(t)+\left(\rho\left(S(t)+V^{T}(t)\right)+(\alpha-\rho) b_{1}(t) N\right) \lambda(t) \\
& +\gamma_{1} a_{2}(t) L^{T} \sigma(t)+\gamma_{2} a_{2}(t) L^{T} \xi(t)=0 \\
& c M \dot{\sigma}(t)+\left(c U^{T}(t)+k b_{1}(t) N_{i j}\right) \sigma(t)+d M \dot{\xi}(t)+d U^{T}(t) \xi(t) \\
& +\gamma_{1} a_{2}(t) L^{T} \dot{\lambda}(t)-\gamma_{1} W(t) \lambda(t)=0 \\
& \\
& d M \dot{\xi}(t)+\left(d U^{T}(t)+\hbar b_{1}(t) N_{i j}\right) \xi(t)+\eta M \dot{\sigma}(t)+\eta U^{T}(t) \sigma(t) \\
& +\gamma_{2} a_{2}(t) L^{T} \dot{\lambda}(t)-\gamma_{2} W(t) \lambda(t)=0
\end{aligned}
$$

\section{Métodos numéricos}

Nesta seção, desenvolveremos três métodos numéricos do sistema de equações diferenciais ordinárias (10) e vamos compará-los.

\subsection{Método numérico acoplado}

Para a primeira equação do sistema, considere a seguinte aproximação de Newmark (Observação: $a^{n}=a\left(t_{n}\right)$, onde $t_{n}$ é o tempo discreto) :

$$
a^{* n}=(1 / 4) a^{n+1}+(1 / 2) a^{n}+(1 / 4) a^{n-1}, \text { onde } a \text { é } \lambda, \sigma \text { ou } \xi
$$

Além disso, para a primeira e segunda derivadas, tomamos as seguintes diferenças centradas no tempo: 


$$
\delta \lambda^{n}=\frac{\lambda^{n+1}-\lambda^{n-1}}{2 \Delta t}, \delta^{2} \lambda^{n}=\frac{\lambda^{n+1}-2 v^{n}+\lambda^{n-1}}{\Delta t^{2}}
$$

Para a segunda e terceira equações, considere a aproximação por Crank-Nicolson:

$$
a^{* n}=(1 / 2) a^{n+1}+(1 / 2) a^{n}, \text { onde } a \text { é } \lambda, \sigma \text { ou } \xi
$$

Além disso, para a primeira derivada, tomamos as seguintes diferenças progressivas no tempo:

$$
\delta \sigma^{n+\frac{1}{2}}=\frac{\sigma^{n+1}-\sigma^{n}}{\Delta t}, \delta \xi^{n+\frac{1}{2}}=\frac{\xi^{n+1}-\xi^{n}}{\Delta t}, \delta \lambda^{n+\frac{1}{2}}=\frac{\lambda^{n+1}-\lambda^{n}}{\Delta t}
$$

Aplicando ao sistema de equações (10), obtemos o seguinte sistema linear de blocos de matrizes:

$$
\mathbf{R} \Psi^{n+1}=-\mathbf{S}_{\mathbf{1}} \boldsymbol{\Psi}^{n}-\mathbf{S}_{\mathbf{2}} \Psi^{n-1}
$$

Usando a recorrência de (15), obtemos os vetores $\Psi^{n+1}=\left[\begin{array}{lll}\lambda^{n+1} & \sigma^{n+1} & \xi^{n+1}\end{array}\right]^{T}$ para $n=1,2, \ldots, N$.

\subsection{Método numérico desacoplado com Preditor-Corretor}

O método com preditor-corretor funciona da seguinte maneira: 1. Achamos normalmente $\lambda^{n+1}$ na 1a. equação, usando como preditores $\xi^{n+1^{P}}=\xi^{n}$ e $\sigma^{n+1^{P}}=\sigma^{n}$. 2 . Resolvemos o sistema da segunda equação com $\xi^{n+1^{P}}=\xi^{n}$ e achamos $\sigma^{n+1}$. 3. Resolvemos o sistema da terceira equação com $\sigma^{n+1}$ e achamos o corretor $\xi^{n+1}$. Dada uma tolerância $\epsilon$, enquanto $\left\|\xi^{n+1}-\xi^{n+1^{P}}\right\|<\epsilon$ e $\left\|\sigma^{n+1}-\sigma^{n+1^{P}}\right\|<\epsilon$, o preditor $\xi^{n+1^{P}}$ recebe o valor de $\xi^{n+1}$, o preditor $\sigma^{n+1^{P}}$ recebe o valor de $\sigma^{n+1}$ e retornamos aos passos 2 e 3 .

\subsection{Método numérico desacoplado}

No método desacoplado, usamos na primeira equação a aproximação de Newmark somente para $\lambda(t)$, além das diferenças centradas para primeira e segunda derivadas. Na segunda e terceira equações, usamos o método de Crank Nicolson de forma análoga ao método acoplado.

\section{Simulação numérica}

Na simulação numérica que fizemos, não temos a solução exata para comparar com a numérica. Por isso, vamos considerar como solução "exata" a solução de uma malha com discretização suficientemente refinada. Vamos considerar esta malha com o número de elementos do espaço $\left(n e l_{y}\right)$ igual a $2^{10}$ e o número de elementos do tempo $\left(n e l_{t}\right)$ igual a $2^{9}$. Em seguida, vamos compará-la com malhas menores para estimar as taxas de convergência e os erros associados às soluções numéricas. 


\section{Exemplo:}

Sejam:

$v(y, 0)=\left(\pi^{2} / 6\right)\left(y^{2}-1\right) ; v^{\prime}(y, 0)=-\left(\pi^{2} / 6\right)\left(y^{2}-1\right) ; \phi(y, 0)=-\left(\pi^{2} / 4\right) \operatorname{sen}\left((\pi / 4)\left(y^{2}-\right.\right.$ $1)) ; \psi(y, 0)=\left(\pi^{2} / 4\right)\left(y^{2}-1\right) ; K(t)=1+t /(2(t+1))$ e as constantes: $\rho=10 ; \alpha=20$; $\gamma_{1}=0,001 ; \gamma_{2}=0,001 ; d=10^{4} ; k=1 ; \eta=10^{4} ; \hbar=1 ; c=2 \times 10^{4}$.

Através da execução do nosso programa feito em MATLAB para o método acoplado, foi encontrada a seguinte tabela de erros e ordens de convergência para $L^{\infty}\left(0, T, L^{2}(-1,1)\right)$ quando $h=\Delta t$ :

Tabela 1: Acoplado - Erros e ordens de convergência - $L^{\infty}\left(0, T, L^{2}(-1,1)\right)$.

\begin{tabular}{|c|c|c|c|c|c|c|}
\hline$h=\Delta t$ & $E_{v}$ & $E_{\phi}$ & $E_{\psi}$ & $p_{v}$ & $p_{\phi}$ & $p_{\psi}$ \\
\hline $1 / 64$ & 0,022414 & 0,000969 & 0,000965 & 1,002032 & 1,819381 & 1,979074 \\
\hline $1 / 128$ & 0,011196 & 0,000237 & 0,000210 & 1,001384 & 2,033097 & 2,199279 \\
\hline $1 / 256$ & 0,005594 & 0,000044 & 0,000033 & 1,000964 & 2,423969 & 2,651475 \\
\hline
\end{tabular}

O tempo de execução para o caso $h=\Delta t=1 / 256$ foi de 164,708 minutos.

Para o método desacoplado com preditor-corretor, temos a seguinte tabela:

Tabela 2: Preditor-Corretor - Erros e ordens de convergência - $L^{\infty}\left(0, T, L^{2}(-1,1)\right)$.

\begin{tabular}{|c|c|c|c|c|c|c|}
\hline$h=\Delta t$ & $E_{v}$ & $E_{\phi}$ & $E_{\psi}$ & $p_{v}$ & $p_{\phi}$ & $p_{\psi}$ \\
\hline $1 / 32$ & 0,000817 & 0,003421 & 0,003805 & 1,819109 & 1,703515 & 1,771199 \\
\hline $1 / 64$ & 0,000229 & 0,000969 & 0,000965 & 1,835780 & 1,819351 & 1,979079 \\
\hline $1 / 128$ & 0,000062 & 0,000237 & 0,000210 & 1,877835 & 2,032367 & 2,198805 \\
\hline
\end{tabular}

O tempo de execução para $h=\Delta t=1 / 256$ e $\epsilon=10^{-6}$ foi de 25,7059 minutos.

Finalmente, para o método desacoplado, temos a seguinte tabela:

Tabela 3: Desacoplado - Erros e ordens de convergência - $L^{\infty}\left(0, T, L^{2}(-1,1)\right)$.

\begin{tabular}{|c|c|c|c|c|c|c|}
\hline$h=\Delta t$ & $E_{v}$ & $E_{\phi}$ & $E_{\psi}$ & $p_{v}$ & $p_{\phi}$ & $p_{\psi}$ \\
\hline $1 / 64$ & 0,000229 & 0,000969 & 0,000965 & 1,835780 & 1,819381 & 1,979074 \\
\hline $1 / 128$ & 0,000062 & 0,000237 & 0,000210 & 1,877835 & 2,033097 & 2,199279 \\
\hline $1 / 256$ & 0,000015 & 0,000044 & 0,000033 & 2,039196 & 2,423969 & 2,651475 \\
\hline
\end{tabular}

O tempo de execução para o caso $h=\Delta t=1 / 256$ foi de 19,7317 minutos. 
Os gráficos para o problema original gerados no caso $h=\Delta t=1 / 32$ foram:

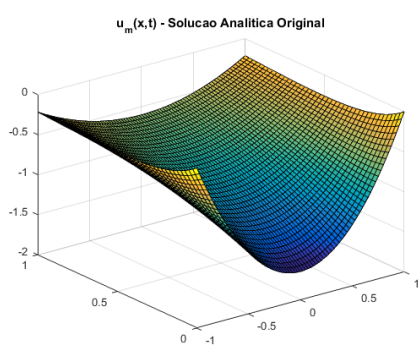

(a) $u(x, t)$

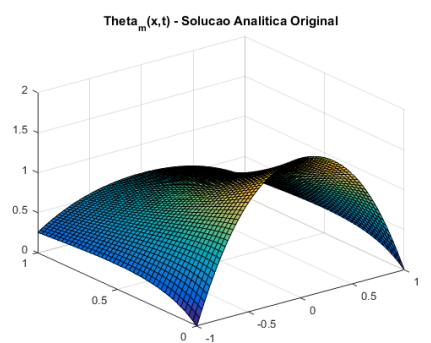

(b) $\theta(x, t)$

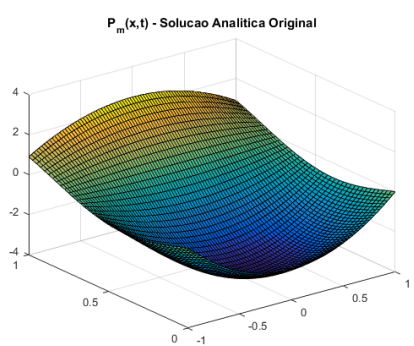

(c) $P(x, t)$

Figura 1: Gráficos - Problema original $-n e l_{x}=64, n e l_{t}=32$.

\section{Conclusões}

No exemplo apresentado aqui, o método acoplado torna-se o mais lento por apresentar uma matriz de tamanho $9 \mathrm{~m}^{2}$ (enorme para valores muito grandes de $m$ ) e ordem linear na convergência de $\lambda^{n}$ para $n=0,1,2, \ldots$ O método numérico desacoplado com preditorcorretor apresentou ordem de convergência quadrática para todas as variáveis do problema, mas foi mais lento que o método desacoplado propriamente dito.

\section{Agradecimentos}

Agradecemos aos professores e alunos do curso de doutorado do Programa de PósGraduação em Informática da UFRJ (PPGI/UFRJ) que colaboraram conosco.

\section{Referências}

[1] M. Aouadi. Theory of generalized micropolar thermoelastic diffusion under lordshulman model. J. Thermal Stresses, (32):923-942, 2009.

[2] M. Aouadi. Qualitative results in the theory of thermoelastic diffusion mixtures. $J$. Thermal Stresses, (33):595-615, 2010.

[3] M. Aouadi. A theory of thermoelastic diffusion materials with voids. ZAMP, (61): 357-379, 2010.

[4] M. Aouadi. Stability aspects in a nonsimple thermoelastic diffusion problem. Appl. Anal., 2012.

[5] B. L. J. Rincon, M.A.; Santos. Numerical method, existence and uniqueness for thermoelasticity system with moving boundary. Comput. Appl. Math., (24):439-460, 2005. 